\title{
The shape of the inner rim in proto-planetary disks ${ }^{\star}$
}

\author{
A. Isella ${ }^{1,2}$ and A. Natta ${ }^{1}$ \\ 1 Osservatorio Astrofisico di Arcetri, INAF, Largo E. Fermi 5, 50125 Firenze, Italy \\ e-mail: isella@arcetri.astro.it \\ ${ }^{2}$ Dipartimento di Fisica, Universitá di Milano, via Celoria 16, 20133 Milano, Italy
}

Received 26 January 2005 / Accepted 29 March 2005

\begin{abstract}
This paper discusses the properties of the inner puffed-up rim that forms in circumstellar disks when dust evaporates. We argue that the rim shape is controlled by a fundamental property of circumstellar disks, namely their very large vertical density gradient, through the dependence of grain evaporation temperature on gas density. As a result, the bright side of the rim is curved, rather than vertical, as expected when a constant evaporation temperature is assumed. We have computed a number of rim models that take into account this effect in a self-consistent way. The results show that the curved rim (as the vertical rim) emits most of its radiation in the near and mid-IR, and provides a simple explanation for the observed values of the near-IR excess (the " $3 \mu \mathrm{m}$ bump" of Herbig Ae stars). Contrary to the vertical rim, for curved rims the near-IR excess does not depend much on the inclination, being maximum for face-on objects. We then computed synthetic images of the curved rim seen under different inclinations; face-on rims are seen as bright, centrally symmetric rings on the sky; increasing the inclination, the rim takes an elliptical shape, with one side brighter than the other.
\end{abstract}

Key words. accretion, accretion disks - radiative transfer - circumstellar matter - planetary systems: protoplanetary disks stars: pre-main sequence - infrared: stars

\section{Introduction}

The structure of the inner regions of circumstellar disks associated with pre-main sequence stars is the subject of intense research. Interferometers working in the near infrared are providing the first direct information on the morphology of disks on scales of fractions of AU. They show that in the majority of cases the observed visibility curves are not well reproduced by flared disk models; rather, they are consistent with the emission of a ring of uniform brightness, of radius similar to the dust evaporation distance from the star (Millan-Gabet et al. 2001; Tuthill et al. 2001).

The interferometric results provide strong support for the idea that the inner disk structure deviates substantially from that of a flared disk because dust evaporation introduces a strong discontinuity in the opacity, which results in a "puffedup" rim at the dust destruction radius, where dust is exposed directly to the heating stellar radiation. The idea of a puffedup inner rim was proposed by Natta et al. (2001) and developed further by Dullemond et al. (2001, hereafter DDN01) for Herbig Ae stars, to account for the shape of the near-infrared excess of these stars (the " $3-\mu \mathrm{m}$ bump"). These authors pointed out that the rim also had the right properties to explain the early interferometric results of Millan-Gabet et al. (2001). Recent theoretical work by Muzerolle et al. (2004) has shown that the condition required to produce a puffed-up inner rim are indeed likely to exist in most Herbig Ae and T Tauri stars. The concept

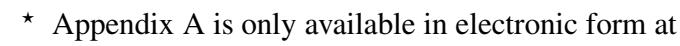
http://www.edpsciences.org of such an inner rim has been widely used to interpret nearIR interferometric data for Herbig and T Tauri stars (Eisner et al. 2004; Muzerolle et al. 2003; Colavita et al. 2003; Eisner et al. 2003; Monnier \& Millan-Gabet 2002; Millan-Gabet et al. 2001). Its effects on the disk structure and emission at larger radii have been discussed by Dullemond \& Dominik (2004), who propose that the classification of Herbig Ae stars in two groups, based on the shape of the far-infrared excess (Meeus et al. 2001), can be interpreted as differences between objects where the outer disk emerges from the shadow of the inner rim and objects where this never happens.

In spite of its success in accounting for a variety of observations, the actual structure of the rim has not been much discussed. DDN01 adopted for their models a very crude approximation, namely that the illuminated side of the rim is "vertical", and that its photospheric height is controlled by radial heat diffusion behind the rim. Such a model, taken at face value, has the obvious disadvantage that the rim emission vanishes for objects seen face-on, for which the projection on the line of sight of the rim surface is null, and for objects seen edge-on, where the rim obscures its own emission. This is clearly inconsistent with observations of the SED, which show that all the Herbig Ae stars with disks have similar near-IR excess, regardless of their inferred inclination (Natta et al. 2001; Dominik et al. 2003).

The vertical shape of the illuminated face of the rim is clearly not physical, as pointed out already by DDN01. Several effects are likely to "bend" the rim: among them, one can expect that radiation pressure on dust grains or dynamical instability, due to self-shadowing effects, could modify the 
illuminated face of the rim (Dullemond 2000; DDN01). None of these suggestions, however, has been explored further.

In this paper, we will discuss in detail a different process, not mentioned so far, which depends exclusively on the basic physics of dust evaporation, i.e., on the dependence of the evaporation temperature on gas density. Circumstellar disks are characterized by a very large variation of the density in the vertical direction, so that the dust evaporation temperature varies by several hundred degrees in a few scale heights; moving vertically away from the disk midplane along the rim, dust will evaporate at lower and lower temperatures, i.e., further away from the central star. This very simple effect curves significantly the inner face of the rim, as we will describe in the following.

The paper is organized as follows. In Sect. 2 we describe the model we use to compute the rim shape and its observational properties. The results are presented in Sect. 3, where we discuss also how the rim depends on dust properties. A discussion of the results, in view of the existing observations, follows in Sect. 4, and a summary is given in Sect. 5.

\section{Model description}

Our model of the inner rim of passive-irradiated flaring disks joins the two different analytical methods to solve the structure of a circumstellar disk proposed respectively by Calvet et al. (1991, 1992, hereafter C92) and Chiang \& Goldreich (1997, hereafter CG97). The temperature in the rim atmosphere is determined using the analytical solution of the problem of the radiation transfer as in $\mathrm{C} 92$, neglecting the heating term due to the mass accretion. The vertical structure of the rim is then computed in a way derived from CG97 and DDN01, adding a relation between the dust vaporization temperature and the gas density as proposed in Pollack et al. (1994). As a result we obtain a curved model for the inner rim whose features are described in the next paragraph.

Although the expressions for the dust temperature derive from a first order solution of the radiation transfer equation, we found (see Appendix) good agreement with the correct numerical result in most cases. To zero order, we can compute the rim structure avoiding proper radiation transfer calculations.

In the limit where the incident angle $\alpha$ of the stellar radiation onto the disk surface is $\alpha \ll 1$, the equations of the temperature for $\tau_{\mathrm{d}}=0$ and $\tau_{\mathrm{d}} \gg 1$ (where $\tau_{\mathrm{d}}$ is the optical depth for the emitted radiation) are formally equal to the optically thin $T_{\mathrm{s}}$ and midplane $T_{\mathrm{i}}$ temperatures introduced by CG97 (two-layer approximation). Therefore, while the two layer approximation is useful to study the structure and the emission features (e.g. silicate features at $10 \mu \mathrm{m}$ ) of the flaring part of the disk (as in DDN01), it must be abandoned in modeling the inner rim, since $\alpha \simeq 1$. Nevertheless we can adapt the CG97 treatment of the vertical structure of the disk to the inner rim using the appropriate expressions for the dust temperature.

In order to clarify this concept and to introduce the relation between the vaporization temperature of dust and the gas density, the basic equations are briefly summarized. We refer to the cited works for a physical discussion of the equations.
We suppose that the disk is heated only by the stellar radiation and we call $\alpha$ the incident angle beetwen the radiation and the disk surface. The incident beam is absorbed exponentially as it penetrates the dusts and if $\tau_{\mathrm{d}}$ is the optical depth for the emitted radiation, the dust temperature $T\left(\tau_{\mathrm{d}}\right)$ is given by the relation $(\mathrm{C} 92)$

$$
\begin{aligned}
T^{4}\left(\tau_{\mathrm{d}}\right)= & T_{\star}^{4}\left(\frac{R_{\star}}{2 r}\right)^{2} \\
& \times\left[\mu(2+3 \mu \epsilon)+\left(\frac{1}{\epsilon}-3 \epsilon \mu^{2}\right) \mathrm{e}^{\left(-\tau_{\mathrm{d}} / \mu \epsilon\right)}\right]
\end{aligned}
$$

where $\mu=\sin \alpha$ and $\epsilon$ is an efficiency factor that characterizes the grain opacity, defined as the ratio of the Planck mean opacity of the grains at the local temperature $T\left(\tau_{\mathrm{d}}\right)$ and at the stellar temperature $T_{\star}$, respectively. In writing the previous equation we have assumed that the scattering of the dust grain is negligible and that the Planck mean opacity is defined as:

$K_{\mathrm{P}}(T)=\frac{\int_{0}^{\infty} B_{v}(T) k_{\nu} \mathrm{d} v}{\int_{0}^{\infty} B_{v}(T) \mathrm{d} v}$.

Following Muzerolle et al. (2003), the continuum emission of the disk is assumed to originate from the surface characterized by the optical depth $\tau_{\mathrm{d}}=2 / 3$.

In the flaring part of the disk, for which $\mu \ll 1$, Eq. (1) can be rewritten in terms of the two layer approximation, proposed in CG97, in which the interior of the disk (with $\tau_{\mathrm{d}} \gg 1$ ) is heated to the temperature $T_{\mathrm{i}}$ by half of the stellar flux, while the other half of the stellar flux is reemitted by the superficial layer heated at the optically thin temperature $T_{\mathrm{s}}$ :

$$
\begin{aligned}
& T^{4}\left(\tau_{\mathrm{d}} \gg 1\right) \simeq T_{\mathrm{i}}^{4}=\frac{\mu}{2}\left(\frac{R_{\star}}{r}\right)^{2} T_{\star}^{4} \\
& T^{4}\left(\tau_{\mathrm{d}}=0\right) \simeq T_{\mathrm{s}}^{4}=\frac{1}{\epsilon}\left(\frac{R_{\star}}{2 r}\right)^{2} T_{\star}^{4} .
\end{aligned}
$$

Following DDN01 we assume that the dust component of the disk is truncated on the inside by dust evaporation, forming an inner hole of radius $R_{\mathrm{in}}$. Inside this hole, only gas may exist but as long as the gas is optically thin to the stellar radiation (Muzerolle et al. 2004) we can neglect its absorbing effect. Near the inner radius $R_{\text {in }}$, since the rim surface is nearly perpendicularly exposed to the stellar radiation $(\mu \simeq 1$ ), Eqs. (3) and (4) must be replaced by the more general relations

$$
\begin{aligned}
& T^{4}\left(\tau_{\mathrm{d}} \gg 1\right) \equiv T_{\infty}^{4}=\mu(2+3 \mu \epsilon)\left(\frac{R_{\star}}{2 r}\right)^{2} T_{\star}^{4}, \\
& T^{4}\left(\tau_{\mathrm{d}}=0\right) \equiv T_{0}^{4}=\left(2 \mu+\frac{1}{\epsilon}\right)\left(\frac{R_{\star}}{2 r}\right)^{2} T_{\star}^{4},
\end{aligned}
$$

derived as limiting cases from Eq. (1).

Since the dust in the rim is by definition close to the evaporation temperature, the value of $\epsilon$ is fixed and depends only on the grain absorbing cross section. Moreover, to first order, since the difference between $T\left(\tau_{\mathrm{d}}=0\right)$ and $T\left(\tau_{\mathrm{d}}=\infty\right)$ is never very large, at the inner radius $(\mu=1)$ the ratio between these two temperature depends only on $\epsilon$ and is given by:

$\frac{T_{0}}{T_{\infty}}=\left[\frac{2 \epsilon+1}{\epsilon(2+3 \epsilon)}\right]^{1 / 4}$. 


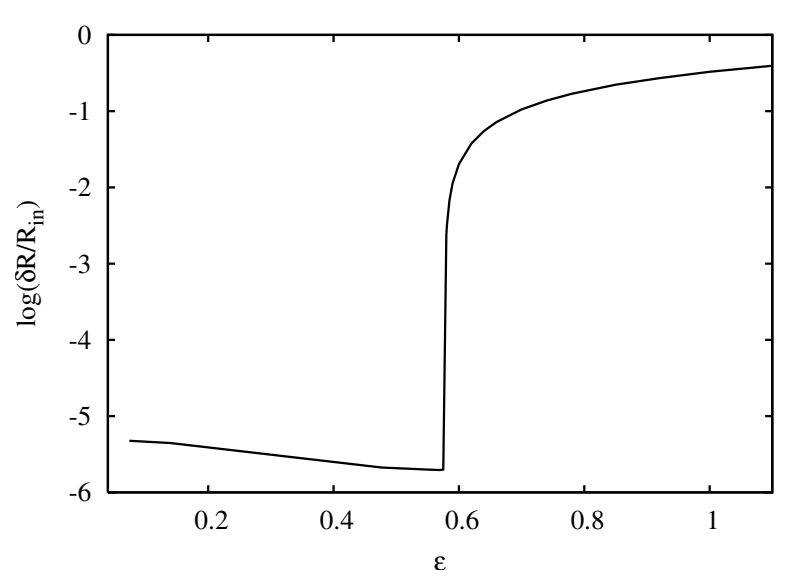

Fig. 1. Width of the region over which $99 \%$ of the stellar radiation is absorbed as function of $\epsilon$.

The rim is defined by the condition that the incoming stellar radiation is entirely absorbed by the intervening dust and in the following we will refer to the surface where $\tau_{\mathrm{s}}=1$ to define its shape and location. Once the grain evaporation temperature $T_{\text {evp }}$ is fixed, one can see from Eq. (1) that the rim has a sharp surface when $\epsilon<\epsilon_{\mathrm{cr}}=1 / \sqrt{3}$. In this case the optically thin temperature $T_{0}$ of the grains is higher than $T_{\infty}$. As soon as a grain can survive, the optical depth for the stellar radiation $\tau_{\mathrm{s}}$ increases rapidly from zero to very high values and the rim surfaces characterized by different values of the optical depth $\tau_{\mathrm{s}}$ are all compressed in a vary narrow zone, located at $R_{\text {in }}$, i.e., where $T_{0}=T_{\text {evp }}$. For $\epsilon>\epsilon_{\mathrm{cr}}$, the dust temperature increases with the optical depth and the transition to optically thick regimes is controlled by the geometrical dilution of the stellar radiation; in this case the transition from $\tau_{\mathrm{s}}=0$ to very high values occurs in a relatively broader region (see Fig. 1); however, also in these cases the rim is located by the $\tau_{\mathrm{s}}=1$ surface with an accuracy better than 10-20\%.

With the assumption that the disk is in hydrostatic equilibrium in the gravitational field of the central star and that is isothermal in the vertical direction $z$, the gas density distribution is expressed by the Gaussian relation

$\rho_{\mathrm{g}}(r, z)=\rho_{\mathrm{g}, 0}(r) \exp \left(-z^{2} / 2 h^{2}\right)$,

where

$\frac{h}{r}=\left(\frac{T_{\infty}}{T_{\mathrm{c}}}\right)\left(\frac{r}{R_{\star}}\right)^{1 / 2}$

defines the relation between the pressure scale $h$ and the interior temperature $T_{\infty}$ at a distance $r$ from the central star. The temperature $T_{\mathrm{c}}$ is a measure of the gravitational field of the central star, expressed by

$T_{\mathrm{c}}=\frac{G M_{\star} \mu_{\mathrm{g}}}{k R_{\star}}$

where $\mu_{\mathrm{g}}$ is the mean molecular weight of the gas.

Note that the interior temperature of the disk $T_{\infty}$ depends on the incident angle $\alpha$ between the stellar radiation and the disk surface, through Eq. (5). The quantity $\mu=\sin \alpha$ accounts for the projection of a disk annulus on a plane perpendicular to the incident radiation and is given by the relation

$\mu=\sin \left(\arctan \left[\frac{r}{z(r)}\right]+\arctan \left[\frac{\mathrm{d} z(r)}{\mathrm{d} r}\right]-\frac{\pi}{2}\right)$,

where $z(r)$ is the axisymmetric equation of the surface of the disk that we want to determine. For the standard flaring model, the incident angle $\alpha$ is given by the relation

$\sin \alpha \equiv \mu \simeq \frac{0.4 R_{\star}}{r}+r \frac{\mathrm{d}(H / r)}{\mathrm{d} r}$,

where $H$ is the photospheric height of the disk, defined as the height to which the optical depth of the disk to the stellar radiation is $\tau_{\mathrm{s}}=1$, on a radially directed ray. For the inner rim we can retain this definition for $H$ and place $z(r)=H(r)$ in Eq. (11), where $H$ is thus given by the relation

$\frac{K_{\mathrm{P}}\left(T_{\star}\right)}{\mu} \int_{H(r)}^{\infty} \rho_{\mathrm{d}}(z, r) \mathrm{d} z=1$.

As shown in DDN01, the ratio $\chi=H / h$, between the photospheric and pressure height, is a dimensionless number of the order of 4-6, depending weakly on the angle $\alpha$, the density $\rho_{\mathrm{d}}$ and the Planck mean opacity of dust at the stellar temperature $T_{\star}$. Finally, to obtain the dust density distribution from Eq. (8) we assume a constant ratio $\rho_{\mathrm{d}} / \rho_{\mathrm{g}}=0.01$.

We can follow CG97 and DDN01 to obtain a self-consistent solution of Eqs. (2), (5), (6), (8), (9), (11), (13) to determine the structure of the disk, as long as dust evaporation can be neglected. When dust evaporation is important, DDN01 have developed an approximate solution of the rim/disk structure under the assumption of constant $T_{\text {evp }}$. The inner rim has a vertical surface toward the star located at the dust evaporation distance $R_{\text {in }}$. The vertical photospherical height $H$ depends on the dust density behind the rim; since the rim is higher than the flaring disk, it casts a shadows over the disk. In this shadowed region, assuming that there is no external heating except the stellar radiation, the pressure height $h$ depends only on the radial heating diffusion. The exact determination of the structure of the rim in this diffusive region would thus require to solve the problem of radiation transport in two dimensions (see Dullemond 2002). Since this goes well beyond our aims, we adopt the approximated relation used in DDN01

$\frac{\mathrm{d}\left(r T_{\infty}^{4}\right)}{\mathrm{d} r} \simeq-\frac{r T_{\infty}^{4}}{h}$

Since $h \propto\left(T_{\infty} R^{3}\right)^{1 / 2}$ (from Eq. (9)), the temperature $T_{\infty}$ can be eliminated and for $h \ll R$ we obtain the relation for $h$ behind the rim

$\frac{\mathrm{d}(h / r)}{\mathrm{d} r}=-\frac{1}{8 R}$.

The solution of Eq. (15) can therefore be used to determine the density $\rho_{\mathrm{d}}(r, z)$ behind the rim through Eq. (8).

We now introduce the relation between the evaporation temperature of the dust and the gas density. The physical reasons for this effect can be easily understood thinking of evaporation as the process by which equilibrium between the gas pressure and the surface tension of the dust grains is 


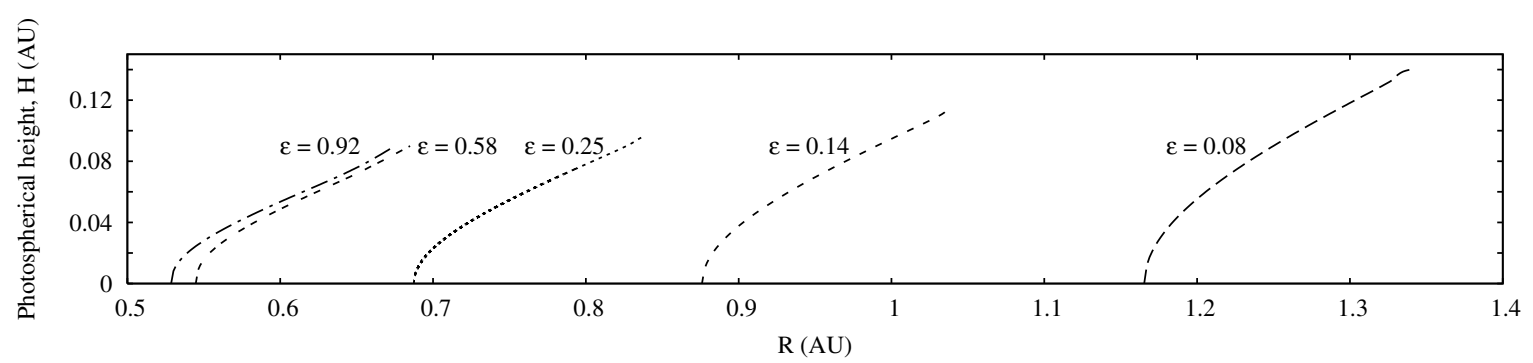

Fig. 2. Photospherical height $H\left(\tau_{\mathrm{s}}=1\right)$ of the inner rim for different values of $\epsilon$, as labelled. The curves have been computed for a star with $T_{\star}=10000 \mathrm{~K}, M_{\star}=2.5 M_{\odot}, L_{\star}=47 L_{\odot}$. Each curve ends where the rim becomes optically thin to the stellar radiation.

reached: the higher the gas density, the higher will be the evaporation temperature. We adopt for the dust in the disk the model proposed by Pollack et al. (1994). In this model, the grains with the higher evaporation temperature are the silicates, which will therefore determine the location of the rim. Their evaporation temperature (see Table 3 in Pollack et al.) varies with the gas density roughly as a power law, of the kind:

$$
T_{\text {evp }}=G \rho_{\mathrm{g}}^{\gamma}\left(r, z_{\mathrm{evp}}\right)
$$

where $G=2000$ and $\gamma=1.95 \times 10^{-2}$. Since $\rho_{\mathrm{g}}$ varies exponentially with $z$ (see Eq.(8)), for $z_{\text {evp }}=h$ the evaporation temperature of dust is only $1 \%$ smaller than that on the midplane, but for $z_{\text {evp }}=H \sim 5 h$ (for a reasonable value of $\chi$ ) the difference is about $20 \%$ and dust evaporation takes place at a larger distance from the star, curving the rim.

To obtain a self-consistent determination of the structure of the curved rim, we implemented a numerical method able to solve Eq. (16) together with the set of Eqs. (2), (5), (6), (8), (9), (11), (13). The distance of dust evaporation in the midplane $R_{\text {in }}$ is computed for $z=0$ in the set of equations and is taken as the starting point of the radial grid on which the rim structure is computed. As a result of the calculations, we obtain the location in the $(R, z)$ plane of the rim surfaces characterized by a constant value of the optical depth. The surface for $\tau_{\mathrm{s}}=0$ is thus the evaporation surface of dust grains, for $\tau_{\mathrm{s}}=1 \mathrm{we}$ obtain the surface relative to the photospheric height $H$, defined through the Eq. (13), while for $\tau_{\mathrm{d}}=\epsilon \cdot \tau_{\mathrm{s}}=2 / 3$ we obtain the emitting surface of the rim.

Moving on the rim surface away from the star, the incident angle $\alpha$ decreases and, when it approaches zero, the determination of the rim shape becomes very difficult. This is mainly due to the fact that the described solution for the radiation transfer neglects the heat diffusion between contiguous annulus of the rim. Therefore both the midplane temperature $T_{\infty}$ and the pressure height $h$ of the rim goes unphysically to zero for $\alpha=0$, according to Eqs. (5) and (9). To avoid this unrealistic behaviour, we use the approximated relation discussed previously (see Eq. (15)) to determine the pressure height $h$ in the region where the diffusion is the dominant heating source. The transition distance between the region of the rim heated by the star and those heated by the diffusion is determined imposing continuity of $\mathrm{d} h / \mathrm{d} r$. The photospherical height $H$ is then determined as for the vertical rim.

\section{Results}

Using the model described in the previous section, we compute the structure of the inner rim for a disk heated by a star with temperature $T_{\star}=10000 \mathrm{~K}$, mass $M_{\star}=2.5 M_{\odot}$ and luminosity $L_{\star}=47 L_{\odot}$. We take a disk surface density $\Sigma(r)=2 \times 10^{3}(R / \mathrm{AU})^{-1.5} \mathrm{~g} \mathrm{~cm}^{-2}$, and a dust-to-gas mass ratio dust/gas $=0.01$. In our models, this value of $\Sigma$ corresponds to a midplane gas density of about $10^{-8} \mathrm{~g} / \mathrm{cm}^{3}$ at $0.5 \mathrm{AU}$ from the star. Note that the results are not very sensitive to the exact value of $\Sigma$, as long as the inner disk remains very optically thick.

The dust properties are those of the astronomical silicates of Weingartner \& Draine (2001). In our models, we consider that all the grains have the same size and characterize their properties with the quantity $\epsilon$, the ratio of the mean Planck opacity at the evaporation temperature to that at the stellar temperature (see Eq. (2)). The evaporation temperature of silicate grains vary from $1600 \mathrm{~K}$, for gas density of $\rho_{\mathrm{g}}=10^{-6} \mathrm{~g} / \mathrm{cm}^{3}$, to $1000 \mathrm{~K}$ for $\rho_{\mathrm{g}}=10^{-16} \mathrm{~g} / \mathrm{cm}^{3}$ (see Eq. (16)). For the Weingartner silicates and a vaporization temperature $T_{\text {evp }} \sim$ $1400 \mathrm{~K}, \epsilon \sim 0.08$ for grains of radius $a=0.1 \mu \mathrm{m}$, and grows to values of about unity for grain radii $>5 \mu \mathrm{m}$.

\subsection{The rim shape}

The shape of the rim is shown in Fig. 2, which plots the locus of $\tau_{\mathrm{s}}=1$ (i.e., the photospheric height $H$ ) as function of $R$ for different values of $\epsilon$. The ending point of the rim $\left(R_{\text {out }}, H_{\text {out }}\right)$ is when the rim becomes optically thin at the stellar radiation.

For $\epsilon \geq \epsilon_{\mathrm{cr}} \sim 0.58$, corresponding to silicate grains bigger than $1.3 \mu \mathrm{m}$, the inner radius $R_{\mathrm{in}}$, the outer radius $R_{\text {out }}$ and the maximum photospheric height of the $\operatorname{rim} H_{\text {out }}$, all vary very little, with values $R_{\text {in }} \simeq 0.50 \mathrm{AU}, R_{\text {out }} \simeq 0.65 \mathrm{AU}$ and $H_{\text {out }} \simeq$ $0.09 \mathrm{AU}$. For smaller values of $\epsilon$, the rim becomes steeper and the inner radius increases. For $\epsilon=0.08$, corresponding to grains with radius $0.1 \mu \mathrm{m}$, the rim has $R_{\text {in }}=1.16 \mathrm{AU}$, $R_{\text {out }}=1.34 \mathrm{AU}$ and $H_{\text {out }}=0.14 \mathrm{AU}$.

The shape of the rim can be roughly characterized by the ratio of its maximum height $H_{\text {out }}$ over width $\Delta R=\left(R_{\text {out }}-R_{\text {in }}\right)$. This quantity, which is nominally infinity in a vertical rim, becomes in the curved rim models $\sim 0.6$ for $\epsilon \gtrsim \epsilon_{\text {cr }}$ and is $\sim 0.8$ for $\epsilon \sim 0.14-0.08$. In other words, as grains grow, the inner rim approaches the star but the bending of the surface varies very little. 


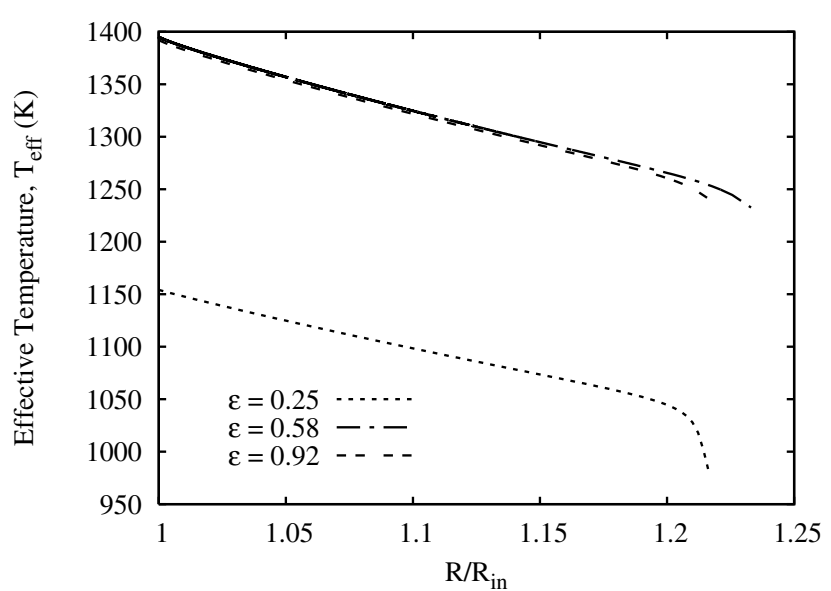

Fig. 3. Effective temperature (i.e., $T\left(\tau_{\mathrm{d}}=2 / 3\right)$ ) along the rim surface for different values of $\epsilon$, as labelled. Each curve is plotted as function of $R / R_{\text {in }}$, where $R_{\text {in }}$ is the distance of the rim from the star on the disk midplane. Note that, in fact, not only $R$ but also $z$ changes along each curve, as shown in Fig. 2. For $\epsilon>\epsilon_{\mathrm{cr}} \simeq 0.58 T_{\text {eff }}$ is almost independent of the grain opacity.

For any given value of $\epsilon$, the dust temperature along the rim is not constant (as in the vertical rim) but decreases from values of about $1400 \mathrm{~K}$, typical of silicate evaporation temperatures at density of $10^{-8} \mathrm{~g} / \mathrm{cm}^{3}$, to $1200 \mathrm{~K}$ for density of $10^{-11} \mathrm{~g} / \mathrm{cm}^{3}$. Figure 3 plots the effective temperature of the rim (i.e., $T\left(\tau_{\mathrm{d}}=\right.$ $2 / 3)$ ) along its surface for different values of $\epsilon$. For $\epsilon \gtrsim \epsilon_{\mathrm{cr}}$ the effective temperature is equal to the vaporization temperature; for $\epsilon<\epsilon_{\mathrm{cr}}$ the effective temperature is generally lower since the dust temperature decreases with the optical depth, according to Eq. (1). This variation, however, is sufficiently small that most of the rim emission occurs in the near-IR, as for the vertical rim.

\subsection{The rim SED}

The fraction of stellar luminosity intercepted by the rim, given by the ratio $H_{\text {out }} / R_{\text {out }}$, varies from $10 \%$, for small values of $\epsilon$, to $14 \%$, for $\epsilon \geq \epsilon_{\mathrm{cr}}$. Assuming that the rim is in thermal equilibrium, the intercepted radiation is equal to the total emitted

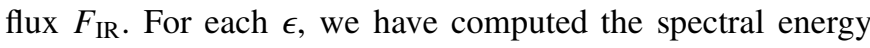
distribution of the rim emission for different inclination angles. As discussed in Sect. 2, the rim emission is computed as that of a blackbody at the local temperature along the rim $\tau_{\mathrm{d}}=2 / 3$ surface. We will come back in the Appendix to this assumption, which, in any case, gives a very good approximation to the global properties of the rim emission (see also Muzerolle et al. 2003, C92). Most of the emission, as expected from the range of temperatures, occurs in the near-IR. We have computed the fraction of the stellar luminosity re-emitted by the rim in the wavelength range $1.25-7.0 \mu \mathrm{m}$ for different values of $\epsilon$ as function of the inclination (Fig. 4). This near-IR excess peaks at zero inclination, where has values between $\sim 10 \%$ (smaller grains) and $\sim 20 \%$ (larger grains). As the inclination increases, the near-IR excess decreases slowly, reaching values between $5 \%$ and $8 \%$, depending on $\epsilon$. For inclination higher then $\sim 80^{\circ}$ the rim emission is self-absorbed. Note that for the

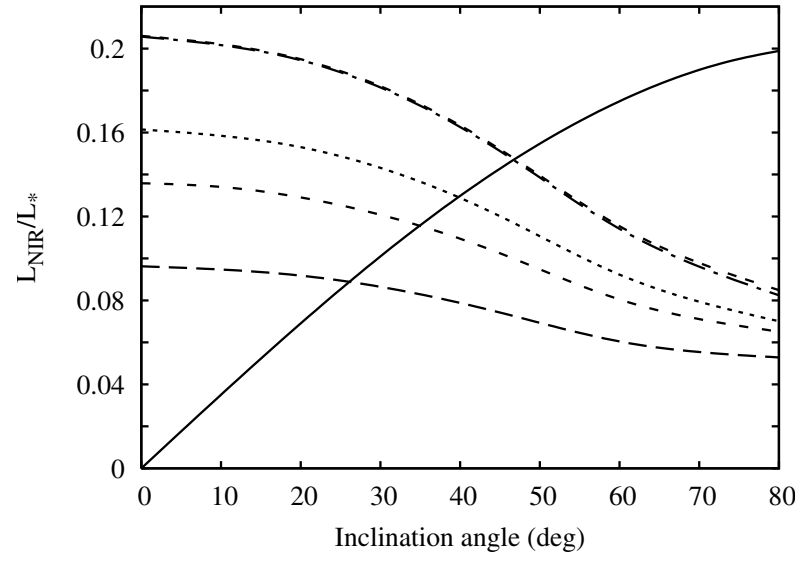

Fig. 4. Near infrared emission of the curved rim (integrated between 1.25-7 $\mu \mathrm{m}$ and normalized to $L_{\star}$ ) for different inclination angles and different values of $\epsilon$. Starting from the bottom, the curves refer to $\epsilon=0.08$ (long-dashed), 0.14 (short-dashed), 0.25 (dotted), $0.58=\epsilon_{\mathrm{cr}}$ (short-dash-dotted), 0.92 (long-dash-dotted). For the last two values of $\epsilon$ the emission is almost the same and the lines overlap. The continuum line plots the emission of the vertical rim, computed for $\epsilon=\epsilon_{\mathrm{cr}}$.

large grains, with $\epsilon \gtrsim \epsilon_{\mathrm{cr}}$, the near-IR excess becomes almost independent of $\epsilon$.

The behaviour described in Fig. 4 is very different from what one obtains in the case of the vertical rim. As described in the previous section, neglecting the dependence of the evaporation temperature of grains from gas density results in the inner face of the rim to be vertical (as in DDN01). With $\epsilon=\epsilon_{\mathrm{cr}}$ and $T_{\text {evp }} \simeq 1400 \mathrm{~K}$ (equal to the evaporation temperature at the inner radius of the curved rim), the inner radius $R_{\text {in }}$ is the same as for the curved rim; the photospheric height, evaluated using the approximation described by Eq. (15), is also the same $\left(H_{\text {out }}=0.09 \mathrm{AU}\right)$; the fraction of stellar luminosity intercepted by the vertical rim is $17 \%$. However, the value of $F_{\text {NIR }}$ observed for different inclination angles is very different, with a very strong (and opposite) dependence on $i$. (Fig. 4). In particular,

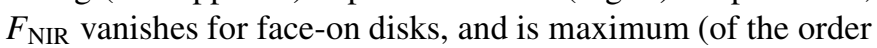
of $20 \%$ ) for very inclined systems, just before self-absorption sets in.

There are also differences between the curved and vertical rim models concerning the shape of the predicted $3 \mu \mathrm{m}$ bump. While the vertical rim has a constant temperature of $1400 \mathrm{~K}$ over all its surface, in the curved rim the temperature varies from $1400 \mathrm{~K}$ on the midplane to about $1200 \mathrm{~K}$ at the outer edge (see Fig. 3) and the SED is broader than a single-temperature black body. In practice, however, this is only a minor effect.

\subsection{Rim images}

Figure 5 shows synthetic images of the curved rim at $2.2 \mu \mathrm{m}$ for grains with $\epsilon=0.08$ (radius of about $0.1 \mu \mathrm{m}$ ) and $\epsilon=$ $\epsilon_{\text {cr }}=0.58$ (radius of about $1.3 \mu \mathrm{m}$ ). As described in Sect. 3.1 (see Figs. 2 and 3), the inner radius of the rim is larger for smaller grains and the surface brightness is lower, due to the lower effective temperature of the emitting surface. 

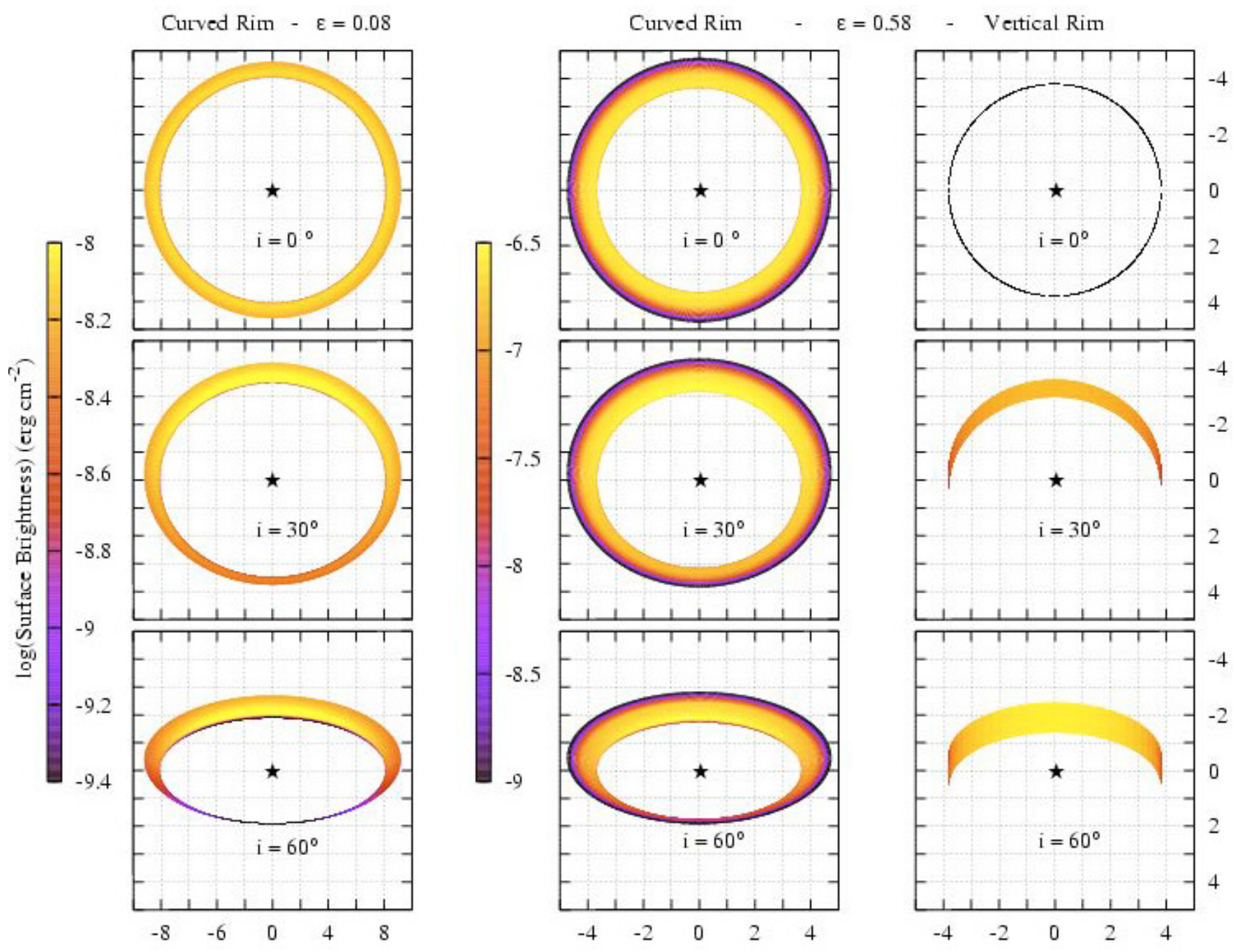

Distance from the central star (milli arcsec)

Fig. 5. Synthetic images of the curved rim for different values of $\epsilon$ and inclination angle ( $i=0^{\circ}$ is face-on); comparison with the vertical rim. The left and central panels show the images of the curved rim calculated for $\epsilon=0.08$ (silicate grains with radius $a=0.1 \mu \mathrm{m}$ ) and $\epsilon=\epsilon_{\mathrm{cr}}=0.58$ $(a=1.3 \mu \mathrm{m})$. The right panels show the images for the verical rim calculated for $\epsilon=0.58$. The surface brightness of the rim, plotted in colors, is computed for a wavelength of $2.2 \mu \mathrm{m}$. The stellar parameters are as in Sect. 3, the distance is $d=144 \mathrm{pc}$. The vertical rim for $i=0^{\circ}$ has zero brightness. Note that as discussed in Sect. 3 for $\epsilon>\epsilon_{\mathrm{cr}}$ the images of the curved rim remain the same.

For the comparison with the vertical model of the rim, the left panels of Fig. 5 show the images of the vertical rim, calculated for $\epsilon=0.58$. The largest difference is at low inclinations: for $i=0^{\circ}$ (face-on disks) the vertical rim vanishes, as the projected emitting surface along the line of sight is zero, while the curved rim has a centrally symmetric ring shape. For a distance of $144 \mathrm{pc}$, the inner radius of the ring, for $\epsilon=\epsilon_{\mathrm{cr}}$, is about 4 milliarcsecond (mas); its brightness peaks practically at $R_{\text {in }}$ $\left(R_{\text {peak }} / R_{\text {in }} \sim 1.05\right)$ and decreases slowly (by about a factor of two) outward, until at $R_{\text {out }}$ it drops to very low values. At this distance, the width of the rim (roughly its FWHM) is about 0.8 mas.

For higher inclination the central symmetry is lost and the projected image is an ellipse with one edge brighter than the other. In general, however, the brightness distribution of the rounded rim is much more symmetric than that of the vertical rim.

\subsection{Grain size distribution}

The results shown so far have been computed assuming that all the grains have the same composition and size. This is in practice unrealistic, and one wonders how the results will change if grains with different properties are present. Different grains absorb differently the stellar radiation and reach very different equilibrium temperatures. The degree of complexity of the radiation transfer problem increases remarkably (see Wolfire \& Cassinelli 1986, 1987) and there is no approximate solution such as Eq. (1). In fact, to the best of our knowledge even $n u$ merical solutions are not available to describe accurately the transition region where some grains are cooler than evaporation and survive while others do not.

We can however estimate the effect of a grain mixture in the following way. Let us consider for simplicity a classical MRN grain size distribution, characterized by a power law $a^{-q}$ 
with $a$ the radius of the dust grains, $q=3.5$, and $a$ varying between a minimum value $a_{\min }=0.01 \mu \mathrm{m}$ and a maximum value $a_{\max } \gg a_{\min }$. From what we have discussed in Sect. 2, if $a_{\max }$ is smaller than $1.3 \mu \mathrm{m}$ (corresponding to $\epsilon=\epsilon_{\mathrm{cr}}$ for the adopted silicate grains), all the grains have a maximum temperature at $\tau_{\mathrm{d}}=0$ and the higher the value of $\epsilon$, the smaller the evaporation distance from the star. As soon as the largest grains can survive, the stellar radiation is rapidly absorbed and all the other grains will also survive. In this case, the shape of the inner rim is controlled by the largest grains in the distribution. The shapes in Fig. 2 and the emitted fluxes shown in Fig. 4 should not change significantly as long as one interprets $\epsilon$ as relative to the largest grains.

The situation is more complex if $a_{\max }>1.3 \mu \mathrm{m}$, since the grains with $\epsilon>\epsilon_{\mathrm{cr}}$ can survive near the star only in an optically thin regime. However, our single-grain models show that there is very little difference in the location and shape of the rim as soon as $\epsilon>\epsilon_{\mathrm{cr}}$. This suggests that varying $a_{\mathrm{max}}$ above the "transition" value (i.e., the value of $\epsilon$ at which the dependence of $T$ on $\tau$ changes from decreasing to increasing) will not change the rim properties any further, at least to zero order.

It is also likely that grains have not just different size but also different chemical composition. In this case, the rim location and properties are determined by the dust species with the highest evaporation temperature, as long as its contribution to the disk opacity is sufficient to make the disk optically thick. As discussed in Sect. 2, in the Pollack et al. (1994) dust model silicates have the highest evaporation temperature. This is why we have performed all our calculations for silicate grains. However, one should keep in mind that, in some dust models, graphite contributes most of the opacity at short wavelengths; since graphite has a much higher evaporation temperature $\left(T_{\text {evp }} \sim 2000 \mathrm{~K}\right)$ than silicates, its presence would move the rim much closer to the central star. Although the details of the rim shape may change, its curving, which is caused only by the dependence of $T_{\text {evp }}$ on the density, will not disappear.

\section{Discussion}

\subsection{The $3 \mu \mathrm{m}$ bump}

The curvature of the inner side of the rim has important consequences on the observable near-IR excess, as shown in Fig. 4, which shows that the predicted near-IR excess ranges between about 10 and $20 \%$, and that there is no significant dependence on the inclination of the disk with respect to the observer. These results compare very well with the existing observations. Figure 6 shows the observed values for a well-studied sample of Herbig Ae stars from Natta et al. (2001) and Dominik et al. (2003); of a total of 16 objects, only one (HD 142527) has $L_{\mathrm{NIR}} / L_{\star}>0.25$, and one (HD 169142) $<0.09$.

The observations of the near-IR SEDs of Herbig Ae stars have also shown that there is no systematic variation of the near-IR excess with the inclination of the disk. The sample of Fig. 6 includes some rather face-on objects such as AB Aur $\left(L_{\mathrm{NIR}} / L_{\star} \sim 0.20, i \sim 20^{\circ}-40^{\circ}\right.$; Fukagawa et al. 2004) and HD $163296\left(L_{\mathrm{NIR}} / L_{\star} \sim 0.21, i \sim 30^{\circ}\right.$; Grady et al. 2000) and some UXORS variable, such as UX Ori, WW Vul and CQ Tau,

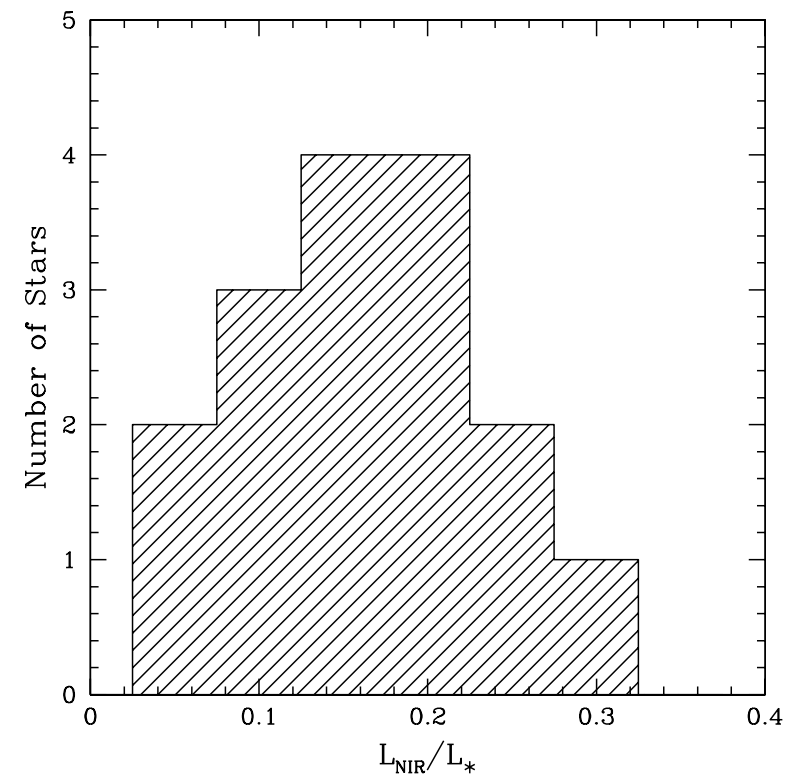

Fig. 6. Histogram of the observed values of $L_{\mathrm{NIR}} / L_{\star}$ for a sample of 16 Herbig Ae stars from Natta et al. (2001) and Dominik et al. (2003). Note that the Dominik et al. values are computed between 2 and $7 \mu \mathrm{m}$, while those from Natta et al. between 1.25 and $7 \mu \mathrm{m}$. The uncertainties are in most cases rather large, due to the subtraction of the photospheric flux, which contributes significantly to the observed ones at short wavelengths, and to variability (see Natta et al. for some examples).

which are generally considered to be close to edge-on, and have values of $L_{\text {NIR }} / L_{\star} \sim 0.12-0.25$. This, which has been a puzzle for the vertical rim, finds a natural physical explanation in our models, which predict that the near-IR excess depends little on the inclination and that, in particular, does not vanish for face-on objects. Actually, our models predict that the largest excesses should be seen in face-on objects, and it would be interesting to explore in detail if this is indeed the case. However, for such a study to be significant, one would need a large sample of objects with known inclinations, which is at present not available.

Another interesting aspect of our results is the dependence of $L_{\mathrm{NIR}} / L_{\star}$ on grain properties. If taken at face-value, one should expect that only objects with relatively large grains (greater than about $1 \mu \mathrm{m}$ ) can have large values of $L_{\mathrm{NIR}} / L_{\star}$, and that low values of the near-IR excess can only occur in face-on objects with small grains. These properties of the rim emission are potentially important for a better understanding of grain properties in the inner disk and should be pursued further in the future, combining observations of disks with well known inclinations with models that explore a broader range of grain properties than we considered in this paper.

\subsection{The rim radius}

One side product of our models is $R_{\text {in }}$, the distance from the star to the rim on the disk midplane. For a face-on disk, $R_{\text {in }}$ practically coincide with the position of the peak of the near-IR brightness. For fixed values of the stellar parameters, $R_{\text {in }}$ depends on the dust properties; its value is shown in Fig. 7 for 


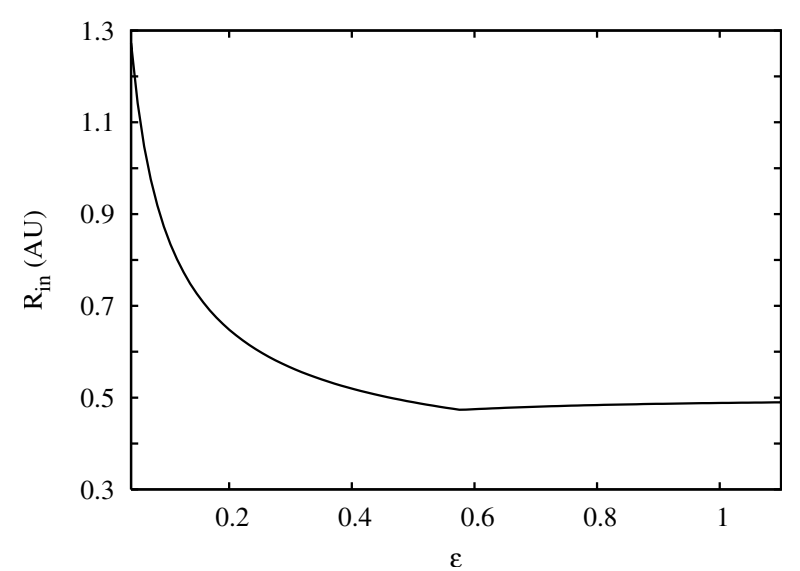

Fig. 7. Behaviour of the inner radius of the rim for different values of $\epsilon$, evaluated for the star and disk parameters described in Sect. 3. Note that for $\epsilon \gtrsim \epsilon_{\mathrm{cr}} \sim 0.58$ (corresponding to silicate grains of $1.3 \mu \mathrm{m}$ ) the radius of the inner rim is almost constant around $0.5 \mathrm{AU}$.

different values of $\epsilon$. Larger grains can survive closer to the star than smaller grains and, because of the inversion in the temperature gradient, $R_{\text {in }}$ does not change much with the grain size once this exceeds the value for which $\epsilon=\epsilon_{\mathrm{cr}}$, so that for $L_{\star}=47 L_{\odot}, R_{\text {in }} \gtrsim 0.5 \mathrm{AU} . R_{\text {in }}$ scales roughly as $L_{\star}^{0.5}$, so that one can expect $R_{\text {in }} \gtrsim 0.1 \mathrm{AU}$ for $L_{\star} \sim 2 L_{\odot}$. These values, especially for small grain sizes, are larger than the predictions of simple, optically thin calculations, because Eq. (1) correctly includes the effect of the diffuse radiation field. Note that if backward scattering cannot be neglected, the temperature at $\tau=0$ will be even higher, increasing further the size of the inner hole (see Appendix).

Values of $R_{\text {in }}$ have been derived for a number of objects from near-IR interferometric observations (see, for example, Akeson et al. 2000, 2002; Millan-Gabet et al. 2001; Monnier \& Millan-Gabet 2002; Colavita et al. 2003; Eisner et al. 2003, $2004,2005)$. The results depend not only on the assumed disk model, but also on other quantities such as the stellar SED, the disk inclination etc., so that a direct comparison with our values of $R_{\text {in }}$ can be misleading. We note, however, that $R_{\text {in }} \sim 0.5 \mathrm{AU}$ for intermediate-mass objects is roughly consistent with the observations, suggesting that in many objects grains have grown to sizes $a \gtrsim 1.3 \mu \mathrm{m}$ (see also Monnier \& Millan-Gabet 2002).

This conclusion, of course, needs to be taken with great caution. There are a number of effects that can change the model-predicted $R_{\text {in }}$, in addition to different grain sizes. For example, on one hand smaller values of $R_{\text {in }}$ can be due to some low-density gas in the inner disk hole, able to absorb the UV continuum from the star (Monnier \& Millan-Gabet 2002; Akeson et al. 2005). Also, the presence of graphite, which has an evaporation temperature of about $2000 \mathrm{~K}$, can decrease $R_{\text {in }}$. On the other hand, the presence of accretion at a significant rate can increase $R_{\text {in }}$ by heating grains in the inner disk to temperatures higher than those produced by the photospheric radiation alone (Muzerolle et al. 2004).

In practice, one needs to compare in detail the model predictions of the quantities measured with interferometers (visibility curves, their dependence on baseline and hour angle, phase measurements) for specific, well-known objects. We are currently performing such a study with the aim of assessing the impact of curved rim models on the interpretation of current (and future) interferometric observations. In particular, we will include a discussion of the effect of the asymmetries of the rim projected images when not face-on. The results will be presented in a forthcoming paper (Isella et al. in prep.).

\subsection{LkHa101}

To the best of our knowledge, the only image of the inner region of a circumstellar disk is that obtained by Tuthill et al. (2001), for LkHa101 using Keck in the $H$ and $K$ bands. The results are reminescent of our images, showing an elliptical ring with a side much brighter than the other. The similarity is very interesting but one should keep in mind that LkHa101 is an early B star with a luminosity between 500 and $50000 L_{\odot}$, depending on its distance. Our model may not apply to such an object (e.g., Monnier et al. 2005).

\section{Summary and conclusions}

In this paper, we discuss the properties of the inner puffed-up rim that forms in circumstellar disks when dust evaporates. The existence of a rim has been claimed starting from the work of Natta et al. (2001), both on theoretical and observational grounds. Here, we investigate the shape of the illuminated face of the rim. We argue that this shape is controlled by a fundamental property of circumstellar disks, namely their very large vertical density gradient, through the dependence of grain evaporation temperature on density. As a result, the bright side of the rim is naturally curved, rather than vertical, as expected when a constant evaporation temperature is assumed.

We have computed a number of rim models that take into account this effect in a self-consistent way. A number of approximations have been necessary to perform the calculations, and we discuss their validity. The basic result (i.e., the curved shape of the rim illuminated face) appears to be quite robust.

For a given star, the rim properties depend mostly on the properties of the grains, and very little on those of the disk itself, for example the exact value of the surface density. The distance of the rim from the star is determined by the evaporation temperature (at the density of the disk midplane) of the dust species that has the highest evaporation temperature, as long as its opacity is sufficient to make the disk very optically thick; in the model of Pollack et al. (1994) of the dust in accretion disks, silicates have the highest evaporation temperature. Therefore, we have assumed in our models dust made of astronomical silicates, and varied their size over a large range of values. We find that the rim properties do not depend on size as soon as $a \gtrsim 1.3 \mu \mathrm{m}$; the values of the rim radii observed with interferometers suggest that in many pre-main-sequence disk grains have grown to sizes of 1 -few $\mu \mathrm{m}$ at least.

The curved rim (as the vertical rim) emits most of its radiation in the near and mid-IR, and provides a simple explanation for the observed values of the near-IR excess (the " $3 \mu \mathrm{m}$ bump" of Herbig Ae stars). Unlike the vertical rim, for curved rims the near-IR excess does not depend much on the inclination, being maximum for face-on objects and only somewhat smaller for 
highly inclined ones. This is in agreement with the apparent similarity of the observed near-IR SED between objects seen face-on and close to edge-on.

We have computed synthetic images of the curved rim seen under different inclinations. Face-on rims are seen as bright, centrally symmetric rings on the sky; increasing the inclination, the rim takes an elliptical shape, with one side brighter than the other. However, the brightness distribution of curved rims remains at any inclination much more centrally symmetric than that of vertical ones. In a forthcoming paper (Isella et al. in preparation) we will discuss the application of the curved rim models to the interpretation of near-IR interferometric observations of disks.

Acknowledgements. We would like to thank Endrik Krügel for having allowed us to make use of his radiation transfer code, Leonardo Testi, Kees Dullemond, Carsten Dominik, Giuseppe Lodato and Giuseppe Bertin for useful discussions. The authors acknowledge partial support by MIUR COFIN grant 2003/027003-001.

\section{References}

Akeson, R. L., Ciardi, D. R., van Belle, G. T., Creech-Eakman, M. J., \& Lada, E. A. 2000, ApJ, 543, 313

Akeson, R. L., Ciardi, D. R., van Belle, G. T., \& Creech-Eakman, M. J. 2002, ApJ, 566, 1124

Akeson, R. L, Walker, C. H., Wood, K., et al. 2005, ApJ, in press

Calvet, N., Patiño, A., Magris, G. C., \& D'Alessio, P. 1991, ApJ, 380, $617 \mathrm{C}$

Calvet, N., Magris, G. C., Patiño, A., \& D’Alessio, P. 1992, Rev. Mex. Astron. Astrof., 24, 27

Chiang, E. I., \& Goldreich, P. 1997, ApJ, 490, 368

Chiang, E. I., Joung, M. K., Creech-Eakman, M. J., et al. 2001, ApJ, 547, 1077

Colavita, M., Akeson, R., Wizinouvich, P., et al. 2003, ApJ, 592, 83
Draine, B. T. 1985, ApJS, 57, 587D

Dominik, C., Dullemond, C. P., Waters, L. B. F. M., \& Walch, S. 2003, A\&A, 398, 607

Dullemond, C. P. 2000, A\&A, 361, L17

Dullemond, C. P., Dominik, C., \& Natta, A. 2001, ApJ, 560, 957

Dullemond, C. P. 2002, A\&A, 395, 853

Dullemond, C. P., \& Dominik, C. 2004, A\&A, 417, 159

Eisner, J. A., Lane, B. F., Akeson, R. L., Hillebrand, L. A., \& Sargent, A. I. 2003, ApJ, 588, 360

Eisner, J. A., Lane, B. F., Hillebrand, L. A., Akeson, R. L., \& Sargent, A. I. 2004, ApJ, 613, 1049

Eisner, J. A., Hillebrand, L. A., White, R. J., Akeson, R. L., \& Sargent, A. I. 2005 [arXiv: astro-ph/0501308]

Fukagawa, M., Hayashi, M., Tamura, M., et al. 2004, ApJ, 605, 53

Grady, C. A. 2000, ApJ, 544, 859

Habart, E., Natta, A., \& Krügel, E. 2004, A\&A, 427, 179

Isella, A., Testi, L., \& Natta, A. 2005, in prep.

Meeus, G., Waters, L. B. F. M., Bouwman, J., et al. 2001, A\&A, 365, 476

Millan-Gabet, R., Schloerb, P. F., \& Traub, W. A. 2001, ApJ, 546, 358

Monnier, J. D., \& Millan-Gabet, R. 2002, ApJ, 597, 694

Monnier, J. D., Millan-Gabet, R., Billmeier, R., et al. 2005, ApJ, in press

Muzerolle, J., Calvet, N., Hartmann, L., \& D’Alessio, P. 2003, ApJ, 597, 149

Muzerolle, J., D’Alessio, P., Calvet, N., \& Hartmann, L. 2004, ApJ, 617,406

Natta, A., Prusti, T., Neri, R., Grinin, V. P., \& Mannings, V. 2001, A\&A, 371, 186

Press, W. H., Teukolsky, S. A., Vetterling, W. T., \& Flannery, B. P. 2002, Numerical Recipes in C++ (Cambrige University Press)

Pollack, J. B., Hollenbach, D., Beckwith, S., et al. 1994, ApJ, 421, 615

Tuthill, P. G., Monnier, J. D., \& Danchi, W. C. 2001, Nature, 409

Weingartner, J. C., \& Draine, B. T. 2001, ApJ, 548, 296

Wolfire, M. G., \& Cassinelli, J. P. 1986, ApJ, 310, 207

Wolfire, M. G., \& Cassinelli, J. P., 1987, ApJ, 319, 850 


\section{Online Material}


A. Isella and A. Natta : Inner disk, Online Material p 2

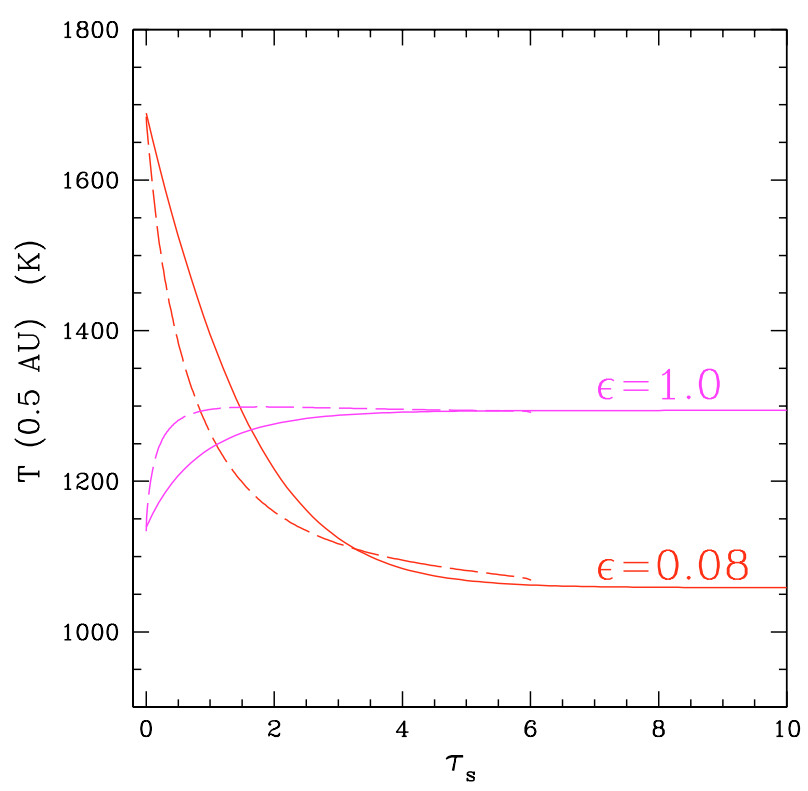

Fig. A.1. Temperature as function of the optical depth to the stellar radiation $\tau_{\mathrm{s}}$ for small grains $(\epsilon=0.08)$ and very large grains $(\epsilon=1)$. The dashed curves are the results of the radiation transfer code, the solid lines the temperature obtained from Eq. (1).

\section{Appendix A}

In this appendix we discuss in detail some of the assumptions on radiation transfer made in building our rim models. We use as templates the results of a radiation transfer code developed by E. Krügel, which is described in Habart et al. (2004). The code considers a plane-parallel slab of dust, illuminated on one side by a star with properties as in Sect. 3 .

\section{A.1. Evaluation of Eq. (1) for single grains}

We show in Fig. A.1 the comparison of the temperature derived from Eq. (1) with the results of the radiation transfer code for two cases, one with $\epsilon=0.08$ (small grains) and one with $\epsilon=1$ (very large grains). In both cases scattering is neglected. The temperature is plotted as function of the optical depth to the stellar radiation $\tau_{\mathrm{s}}$. One can see that Eq. (1) gives the correct values of $T$ for $\tau_{\mathrm{s}}=0$ and for $\tau_{\mathrm{s}}=\infty$. For intermediate values of $\tau_{\mathrm{s}}$, the results are in both cases accurate within $10 \%$. This is more than adequate for the purposes of this paper.

\section{A.2. SED}

We compare now the SED of a black-body at temperature $T\left(\tau_{\mathrm{d}}=2 / 3\right)$ from Eq. (1), adopted in this paper to describe the rim emission (see Sect. 3.2) to the results of the radiation transfer code (dashed curves) for the same grains of Fig. A.1. The approximation is very good for large grains. For small values of $\epsilon$, the discrepancy is larger, and, in particular, one cannot reproduce any dust feature. However, also in the case $\epsilon=0.08$, the difference in $L_{\mathrm{NIR}} / L_{\star}$ is only of $4 \%$.

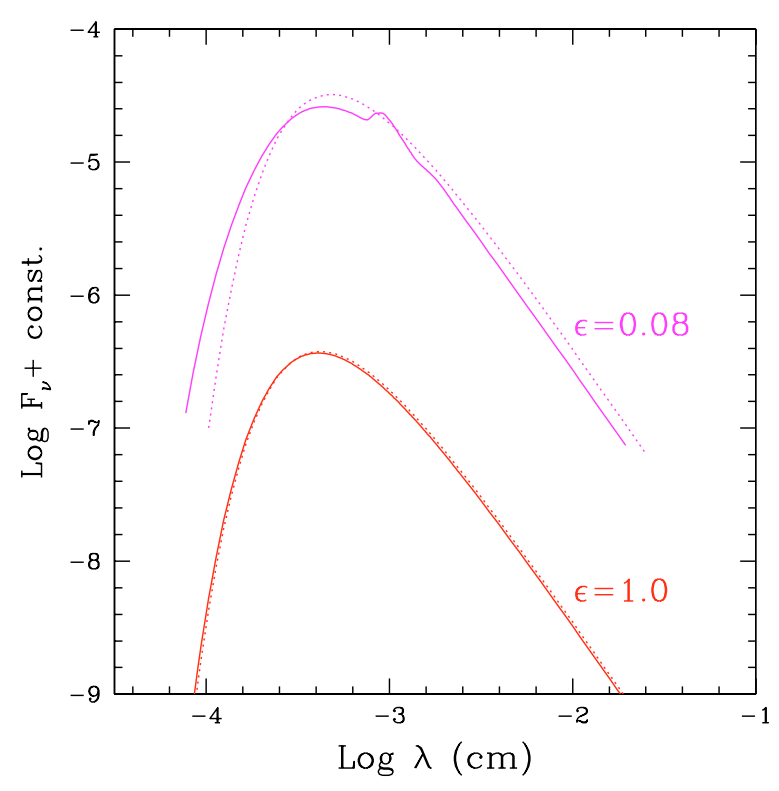

Fig. A.2. Comparison of the SED computed with the radiation transfer code (dotted lines) and as a black-body at $T\left(\tau_{\mathrm{d}}=2 / 3\right)$ from Eq. (1) (solid line) for $\epsilon=0.08$ and $\epsilon=1.0$, as labelled.

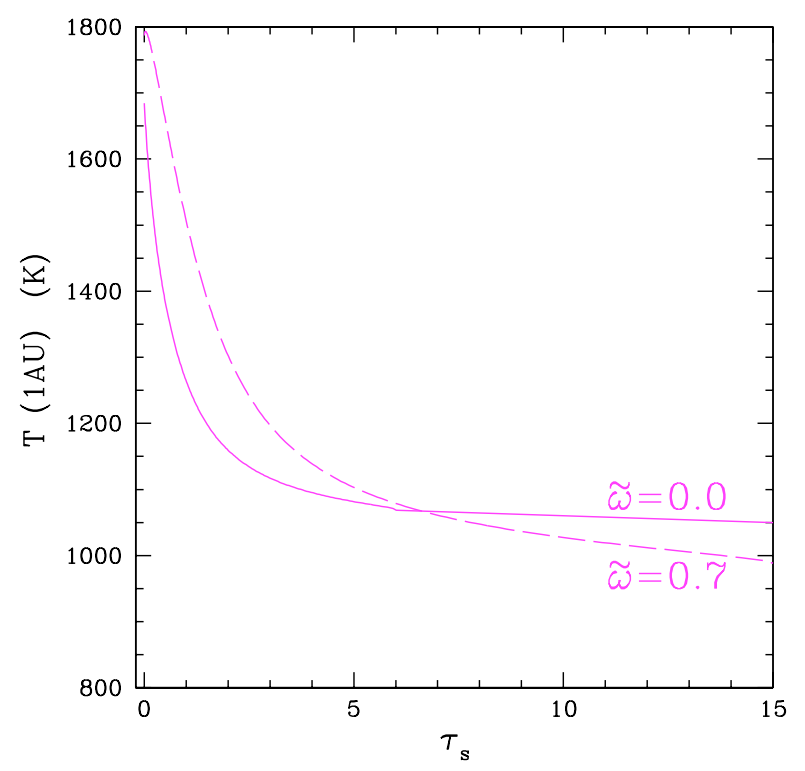

Fig. A.3. Temperature profile for grains with $\epsilon=0.08$, when scattering is included ( $\tilde{\omega}=0.7$; dashed line) or suppressed ( $\tilde{\omega}=0.0$; solid line).

\section{A.3. Scattering}

Scattering has the obvious effect of increasing the grain temperature at the slab surface and decreasing it at large optical depth. We compare in Fig. A.3 the temperature profile of grains with $\epsilon=0.08$ when scattering is properly included in the radiation transfer (dashed line) rather than suppressed $\left(Q_{\mathrm{sc}}=0\right.$.). The curves are labelled with the values of $\tilde{\omega}$, defined as $Q_{\mathrm{sc}}(1-g) / Q_{\mathrm{sc}}(1-g)+Q_{\mathrm{abs}}$, where $g$ measures the asymmetry of the scattering phase function; $\tilde{\omega}=0$ if $Q_{\mathrm{abs}}=0$ or if the scattering is forward peaked $(g=1)$. All quantities are averaged over the stellar radiation field and the corresponding SEDs show that the value of $L_{\mathrm{NIR}} / L_{\star}$ decreases by about $25 \%$ when scattering is included. 